\title{
Contributions to the acoustic excitation of bubbles released from a nozzle
}

\author{
Helen Czerski ${ }^{\text {a) }}$ and Grant B. Deane \\ Marine Physical Laboratory, Scripps Institution of Oceanography, La Jolla, California 92093-0238
}

(Received 24 September 2009; revised 5 August 2010; accepted 9 August 2010)

\begin{abstract}
It has recently been demonstrated that air bubbles released from a nozzle are excited into volume mode oscillations by the collapse of the neck of air formed at the moment of bubble detachment. A pulse of sound is caused by these breathing mode oscillations, and the sound of air-entraining flows is made up of many such pulses emitted as bubbles are created. This paper is an elaboration on a JASA-EL paper, which examined the acoustical excitation of bubbles released from a nozzle. Here, further details of the collapse of a neck of air formed at the moment of bubble formation and its implications for the emission of sound by newly formed bubbles are presented. The role of fluid surface tension was studied using high-speed photography and found to be consistent with a simple model for neck collapse. A re-entrant fluid jet forms inside the bubble just after detachment, and its role in acoustic excitation is assessed. It is found that for slowly-grown bubbles the jet does make a noticeable difference to the total volume decrease during neck collapse, but that it is not a dominant effect in the overall acoustic excitation.
\end{abstract}

(C) 2010 Acoustical Society of America. [DOI: 10.1121/1.3484087]

PACS number(s): 43.30.Nb [KGF]

Pages: 2625-2634

\section{INTRODUCTION}

Bubbles in the open ocean play a central role in a number of boundary layer processes, such as enhanced air-sea gas flux, ocean albedo, the production of marine aerosols and the generation of breaking wave noise. Despite their importance, quantifying bubble production rates in the upper ocean remains a difficult problem. The potential exists to infer bubble creation rates in breaking waves using the pulse of sound radiated by each bubble at the moment of formation.

It has been known for many years that air bubbles in water produce sound when excited into breathing mode oscillations. These oscillations arise naturally in many environments, and are the source of the sound of running water, ${ }^{1}$ breaking waves ${ }^{2}$ and rainfall on deep water. ${ }^{3}$ Bubbles behave acoustically like high Q oscillators, and at the moment of their formation they emit a narrow-band pulse of sound that has the form of an exponentially decaying sinusoid. Analysis of the radiated sound shows that the most important nonequilibrium conditions for exciting the breathing mode oscillations that drive the acoustic emission occur in the first few oscillation cycles of the bubble. ${ }^{4}$ The excitation of this breathing mode is intimately linked with the dynamics of bubbles as they are formed, and several theories have been suggested for the excitation mechanism. While considering the bubbles formed by raindrop impact, Longuet-Higgins ${ }^{5}$ suggested three mechanisms, which were discussed further by Pumphrey and Ffowcs Williams ${ }^{6}$ in the context of bubbles formed underneath breaking waves. The first was the increased pressure on closure due to surface tension, the Laplace pressure. The second was a resonance between shape mode oscillations (excited by the distortion of the

\footnotetext{
${ }^{a)}$ Present address: Graduate School of Oceanography, University of Rhode Island, Narragansett, RI 02882.
}

bubble at the moment of formation) and the breathing mode oscillations. ${ }^{7,8}$ The third was the effect of the fluid flow around the bubble at the moment of formation. Hollet and Heitmeyer ${ }^{9}$ added another to this list- the effect of the additional hydrostatic pressure if the bubble is formed a significant distance beneath the surface. More recently, Manasseh $^{10,11}$ examined high-speed photographs of a bubble forming from a nozzle and simultaneously recorded the acoustic emission. He concluded that an increase in gas pressure inside the bubble caused by the sudden formation of an internal fluid jet just after pinch-off could stimulate breathing mode oscillations. ${ }^{12}$ A recent contribution ${ }^{4}$ has been the development of a simple model for the collapse of the bubble neck, showing that the rapid volume change as the neck retracts can account for most of the forcing required to stimulate the observed acoustic pulse. Here, we extend that investigation and consider the role of surface tension and the internal jet of water formed by neck collapse on the excitation of breathing mode oscillations.

Bubbles in the open ocean are undoubtedly formed as the result of a variety of hydrodynamical processes, including turbulent fragmentation within wave crests, ${ }^{13}$ drops, Mesler entrainment and perhaps other unknown processes. Here, we consider a bubble released slowly from an underwater nozzle, which we are using as a simple model system for bubble acoustical excitation. As a bubble is growing at a nozzle, the surface tension in the bubble walls dominates the bubble buoyancy, keeping the bubble attached to the nozzle until the configuration becomes unstable. ${ }^{14}$ At this point, a neck forms and increasingly rapidly collapses radially inwards with an approximately hyperbolic profile. At the moment of pinch-off, the part of the air bubble closest to the nozzle is almost conical, and once the bubble has pinched off, this conical neck remnant collapses upwards toward the center of the bubble. Bubbles emerging from nozzles have 


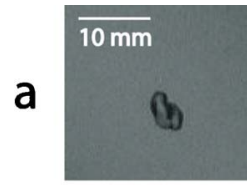

(i) $t=0$

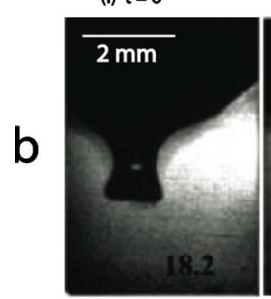

$$
\text { (i) } t=0
$$



(i) $\mathrm{t}=\mathbf{0}$

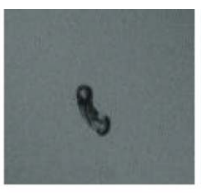

(ii) $t=4 \mathrm{~ms}$



(ii) $\mathrm{t}=510 \mu \mathrm{s}$

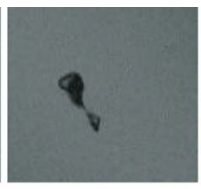

(iii) $\mathrm{t}=8 \mathrm{~ms}$

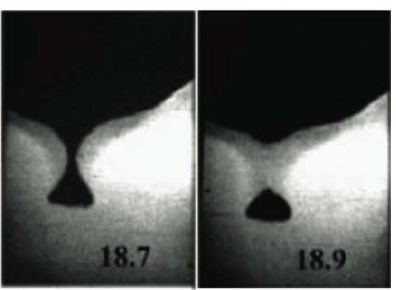
(iii) $t=64 \mu \mathrm{s}$



(iii) $\mathrm{t}=910 \mu \mathrm{s}$

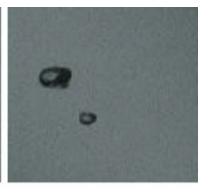

(iv) $\mathrm{t}=12 \mathrm{~ms}$ (iv) $\mathrm{t}=90 \mu \mathrm{s}$

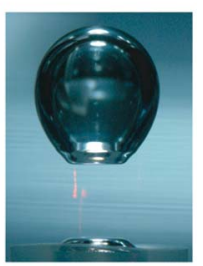

(iv) $t=2260 \mu \mathrm{s}$
FIG. 1. (Color online) Examples of neck formation and collapse as bubbles are formed. (a) A bubble fragmenting in turbulent flow. (b) A bubble formed at the bottom of a crater caused by the impact of a liquid drop, reproduced from Elmore et al. (Ref. 18). (c) The formation of a bubble from a nozzle (the nozzle is just visible at the bottom of the frame). In all three cases, the formation and pinch-off of a neck of gas and the subsequent rapid retraction of the neck remnant can be seen. All the times given are relative to an arbitrary reference.

been studied by many authors, but the main focus has been the dynamics leading up to the moment of pinch-off, ${ }^{12,15,16}$ and experimental issues. ${ }^{17}$ Because the majority of the acoustic excitation occurs after pinch-off, most of these studies are not directly relevant to bubble acoustic excitation.

\section{A. The role of neck collapse in acoustic excitation}

At the moment of formation, bubbles are necessarily non-spherical. Surface tension forces drive the bubble toward sphericity once it is separated from the parent body of gas, but it is the very early stages that are of most interest to acoustic studies. Bubbles pinch off from another body of gas as a result of a variety of processes (fragmentation, falling drops, etc.), but the formation and collapse of a neck which then pinches off and collapses back toward the center of the new bubble seems to be a common factor. Figure 1 shows examples of this in three different situations: bubbles fragmenting in turbulence, bubbles caused by falling drops and bubbles pinching off from nozzles. In all three, the formation of a neck and the rapid retraction of the neck remnant after pinch-off can be seen. In this paper we will focus on the dynamics of bubbles formed at nozzles, but we expect that the same acoustic forcing mechanism may also be dominant for bubbles formed in other situations.

Deane and $\mathrm{Czerski}^{4}$ demonstrated that the volume change caused by the collapse of the conical neck remnant could account for the necessary acoustic forcing for a bubble formed at a nozzle. In this paper, we present data to partially answer some of the outstanding questions about the mecha- nism driving these bubbles into breathing mode oscillations. We examine bubbles in water with surfactant added, to further test the assertion that surface tension is driving the neck collapse. We have also photographed the internal jet produced as a bubble pinches off from a nozzle and have estimated how its volume changes with time. This has allowed us to assess its importance in acoustic forcing relative to the simple conical neck collapse model alone, for slowlyreleased bubbles.

\section{EXPERIMENTAL METHODS}

We chose to examine bubbles emerging slowly from a vinyl nozzle in a small tank since this is a simple reproducible system and does not require equipment such as flashes and cameras to be mounted underwater. The experimental configuration was the same as for Deane and Czerski. ${ }^{4}$ The nozzle was positioned vertically at the base of a small transparent tank, and gas was supplied at a very low flow rate through a regulator so there was a gap of at least three seconds between successive bubbles. As the growing bubble became unstable close to the time of pinch-off, its neck narrowed rapidly and moved out of the path of a laser beam, allowing laser light to reach a photodiode on the opposite side of the tank. This provided a reliable trigger for the flashes and the data acquisition system. The details of the bubble shape were captured using strobe photography with a $20 \mu$ s flash duration. The strobe lights used were manufactured by Shimpo, model DT 314. A hydrophone (model International Transducer Corporation 1089D) was also positioned close to the bubble (1-2 cm away) in order to collect acoustic data with the same time reference as the strobes. The hydrophone signal was amplified using an Stanford Research Systems SR 560 low noise preamplifier. The acoustic traces from the hydrophone were used to provide a context for bubble acoustic forcing at different stages of neck collapse, but were not used for a quantitative analysis, thus avoiding any concerns about tank reverberation. Tank reverberation will alter the damping rate of the breathing mode oscillations $^{19}$ and the proximity of the tank walls alters the bubble natural frequency, ${ }^{20}$ but neither will significantly alter the initial conditions forcing bubble radiation during neck collapse.

Figure 2 shows the setup used. The nozzle was positioned approximately $5 \mathrm{~cm}$ from the bottom of a transparent acrylic tank with a $13 \mathrm{~cm}$ square cross-section. The nozzle mouth was usually $10 \mathrm{~cm}$ below the surface of the water, and we have ignored any additional pressure due to the bubble depth. Bubbles rose from the nozzle to the surface in less than half a second and a new bubble was produced approximately every three seconds, so there was only ever one bubble in the tank at a time. Care was taken to keep the interior of the tank clean and free from surfactants (except when they were added deliberately). The pressure of the supplied air could be varied in order to change the rate of bubble production. In all our experiments, the bubble rate sufficiently low that at pinch-off the bubble was very close to quasi-static, and consecutive bubbles did not interfere with each other. Most of the experimental data discussed here was 


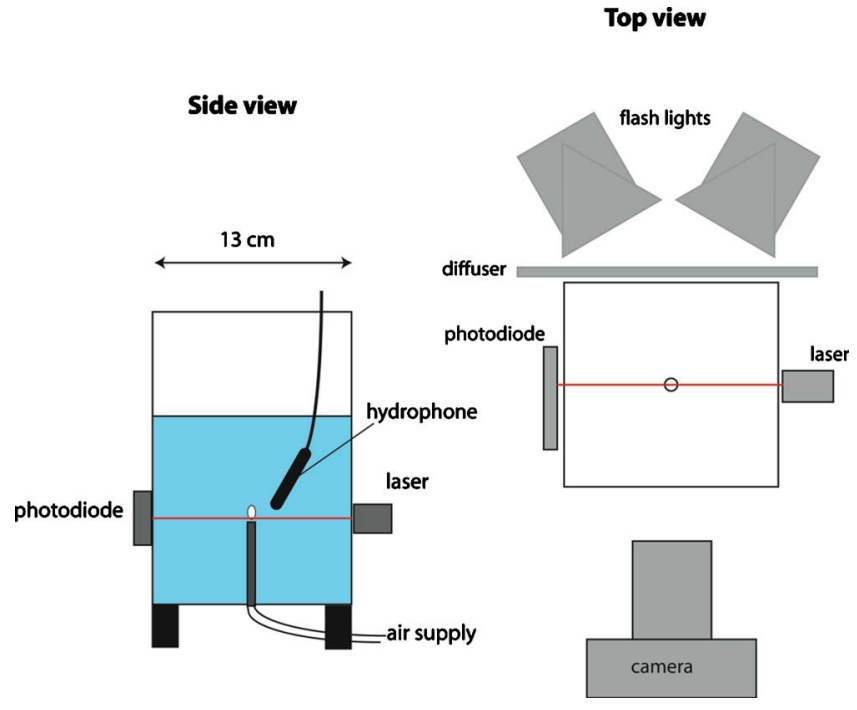

FIG. 2. (Color online) A schematic diagram of the experimental set-up. A bubble formed at the top of a nozzle and the acquisition system was activated when it pinched off. As the bubble neck narrowed, it moved out of the path of the laser beam and resulting rise in the photodiode signal was used as the trigger for the flash lights and data collection.

collected from bubbles generated using a transparent plastic nozzle. The transparent material allowed light to reach the bubble from below, so that the internal jet could be imaged. A plastic nozzle with external and internal diameters of 6.0 $\mathrm{mm}$ and $3.3 \mathrm{~mm}$ produced bubbles with a width of $4.1 \mathrm{~mm}$ at the moment of pinch-off. A metal nozzle with external and internal diameters of $2.1 \mathrm{~mm}$ and $1.6 \mathrm{~mm}$ produced bubbles of a very similar size, but was much more challenging to use because the pinch-off point was very close to the nozzle mouth, and interfered with the laser trigger.

Two strobe lights could be positioned anywhere around the outside of the tank, and diffusers were used to prevent direct reflections into the camera. To produce silhouette images, they were directly behind the bubble, facing into the camera. In order to photograph the jet inside the bubble, more care with lighting was required and mirrors positioned inside the tank were used to provide illumination from many directions. The strobes could be triggered independently with any chosen delays, double-exposing a single frame and allowing the change in shape of the bubble to be followed very accurately with time. The strobes were triggered with pulses from a National Instruments DAQ board with a Labview interface, so that precise timing of the pulses was easily controlled. There was a delay of approximately $40 \mu$ s between triggering the strobes and the start of light emission, and this was taken into account when the time relative to the acoustic trace was calculated. By triggering two flashes with a known delay between them, the relative time difference between the two images on a single exposure was very accurately known (to within a microsecond). The experiment was surrounded by blackout material and photographs were taken using a digital single lens reflex camera with a long exposure time.

Figure 3 shows an example of a double strobe image, typical of the ones used to measure how the position of the base of the bubble varied with time. The first exposure was as close to the point of pinch-off as could be resolved, and

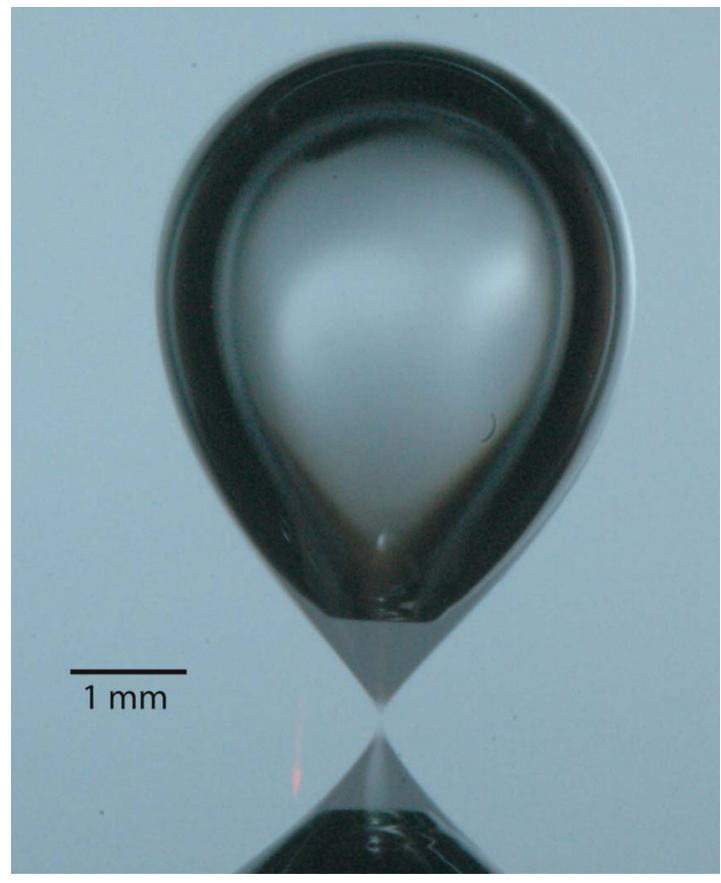

FIG. 3. (Color online) An example of a double strobe image in silhouette. The first exposure was very close to the moment of pinch-off (the small cylindrical part of the neck where pinch-off occurs is faint and blurred) and the second one was $700 \mu$ s later. The light gray area is from the first exposure and the darker area is where the two bubble images are overlaid, so the edge of the dark region shows the shape of the bubble at the later time. The top of the nozzle is just below the field of view, but the symmetry of the collapse is evident. It can be seen that the "uncollapsed" conical walls have moved radially inwards slightly between the two exposures.

the second was at the time of interest. Direct estimations of the velocity could be made by using a shorter inter-flash time and triggering the flash pair at different times after pinch-off. Only one frame was recorded for each bubble, so data from many successive bubbles was used to form each plot. The progression of the bubble shape with time was found to be very reproducible, and no differences could be identified between photographs of different bubbles taken at the same time relative to the moment of their formation. An image subtraction technique was used to confirm that fewer than one quarter of one percent of the pixels differed between photographs taken at similar times relative to pinch-off.

For the first $500 \mu \mathrm{s}$, the top of the bubble did not move detectably relative to the nozzle. After that, it did move upwards slowly and the results were corrected for this. The top half of the bubble remained the same shape during the neck collapse-no deviation could be detected in the first millisecond. The model we propose is only valid for the first five hundred microseconds or so, and so we ignore buoyancy in the discussion here.

\section{THE MODEL FOR NECK COLLAPSE}

A simple model was used to predict the rate of the neck collapse, in order to make a comparison with the measured results. This is described in Deane and Czerski, ${ }^{4}$ but we reproduce the details here for completeness. The shape of the bubble at pinch-off is shown in Fig. 4. The top of the bubble is similar in shape to a prolate spheroid and the lower part of 


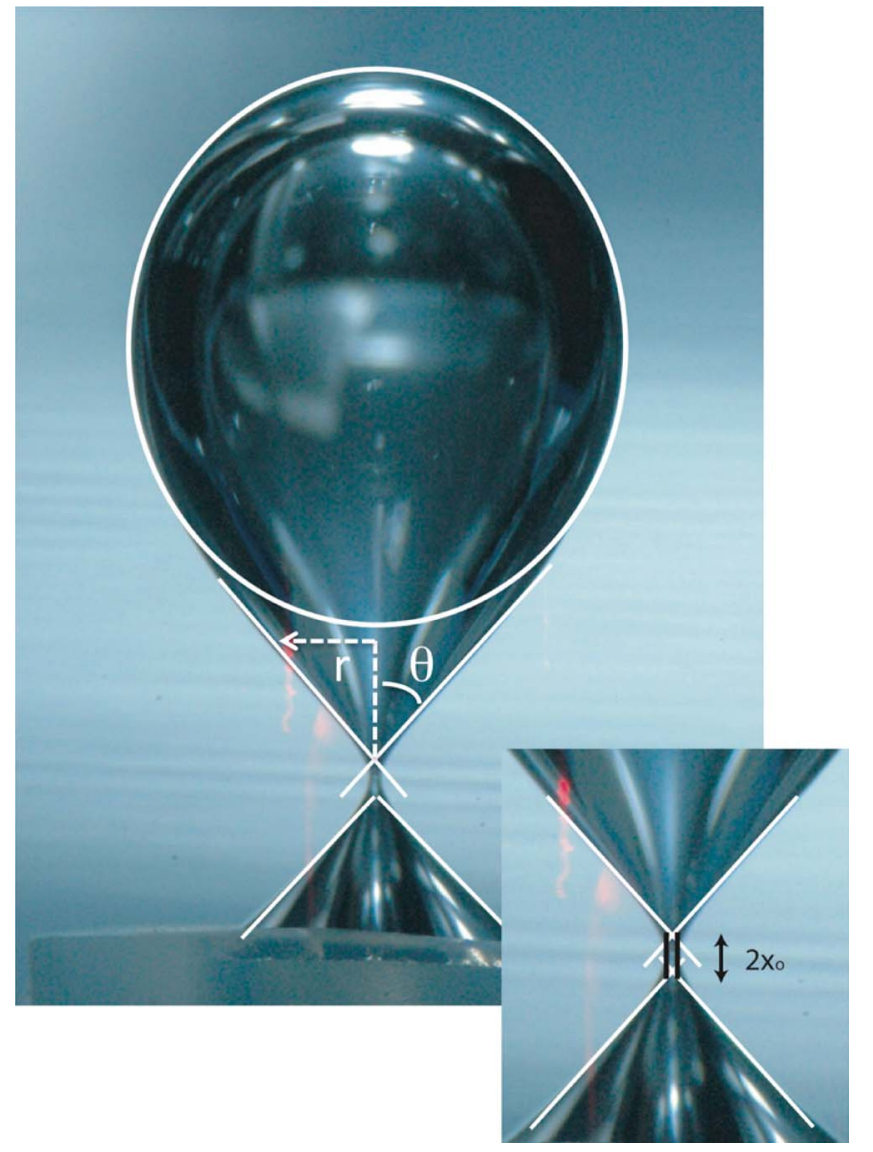

FIG. 4. (Color online) Bubble geometry. We assumed that the lower part of the bubble could be modeled as a cone with a small cylinder at the base. The inset shows how the length of the cylindrical part of the neck was estimated. Two parallel lines were drawn along the walls of the narrowest part of the neck. The point at which these lines intersected the lines describing the cone as considered to be the end of the neck.

the bubble is close to being conical with a half-angle of $39^{\circ}$, except for a small region close to the pinch-off point. At the base of the cone at pinch-off, there is a very narrow thread of approximately $0.13 \mathrm{~mm}$ in length from the center of the neck to the start of the cone (for a $4 \mathrm{~mm}$ diameter bubble). The collapse of this thread is very rapid and the flash duration (20 $\mu \mathrm{s})$ is longer than the collapse time. We model this thread as a cylinder-its exact shape is not crucial since its volume is so small relative to the collapsing cone volume. The coordinate origin is the center of the symmetrical neck joining the bubble to the bulk gas supply, and $x$ is vertical distance. We assume that the bubble is axisymmetric and $r$ is the radial distance of the bubble wall from the central axis. We assume that the cylindrical thread has a radius $r_{o}$ and a length $x_{o}$, and that at $x>x_{o}$ the bubble is conical.

The following analysis applies only to the early part of the cone collapse, which is driven by surface tension forces. This force initially accelerates the surrounding water to fill the space where the neck was. This water then has momentum and carries on moving in the same direction, inwards toward the bubble center. If it continues to move forward to form a jet, extra surface is created with an associated energy expenditure, so some of the kinetic energy is transferred back to surface tension energy while the rest is used to compress gas inside the bubble or is dissipated. This internal pressure change (caused by the forced volume decrease of the bubble in the first $500 \mu$ s after pinch-off) is the driving mechanism for the breathing mode oscillations of the bubble. The initial rarefaction in the acoustic pulse is due to the rapid inward flow of water, and the subsequent oscillations are the response of the bubble to the sudden internal pressure increase caused by that inward flow.

The exact mechanism deserves careful consideration, because the acoustic propagation caused by the inward collapse of the neck of air is associated with an unusual relationship between the bubble wall displacement, velocity and acceleration when compared with the damped harmonic oscillator. First of all, we note that the expression which describes the pressure radiated from the bubble (Ref. 21, Eq. 3.128) depends on the bubble wall acceleration only, and no assumptions about simple harmonic oscillation are made during its derivation. The pressure is given by

$$
P_{b}(t) \approx \rho \frac{R_{0}^{2}}{r} \ddot{R}
$$

where $R_{0}$ is the bubble equilibrium radius, $\rho$ is fluid density, $R$ is the current bubble radius, dots indicate the derivative with respect to time, $P_{b}$ is the acoustic pressure radiated by the bubble and $r$ is the radial coordinate of the point of interest.

During simple harmonic oscillation, $R \propto-\ddot{R}$, and so at the points in the oscillatory cycle when $R>R_{0}$ (during both bubble expansion and compression), a rarefaction is propagating outward. However, the case of bubble pinch-off described in this paper is different. The pinch-off dynamics cause initial conditions which are not possible during simple harmonic motion.

We will base our examination of the bubble oscillations after pinch-off on the assumption that the radiated pressure can be described by considering a spherically symmetric bubble. We justify this by noting that the wavelength of radiated sound is far larger than the bubble size (indicating that the consideration of the volume oscillation is sufficient), and also that the mathematics, physics and observations resulting from this assumption are all consistent. A full numerical analysis is beyond the scope of this paper.

We will consider the equivalent spherical radius $R_{e q}$, so that the bubble volume $V$ is proportional to $R_{e q}{ }^{3}$. The volume change (described in Ref. 4 and also in Eq. (6)) is approximately given by $\Delta V(t)=-\Phi t^{2}$. Consequently, $\dot{V}=-2 \Phi t$ and $\ddot{V}=-2 \Phi$ where $\Phi$ is a positive constant. Therefore, just after the moment of bubble pinch-off, $R_{e q}, \dot{R}_{e q}$ and $\ddot{R}_{e q}$ are all negative. This would not occur during simple harmonic oscillation. This leads to two effects. First, since $\ddot{R}_{e q}$ is negative, a rarefaction will propagate outwards as the neck of air collapses. Second, since $R_{e q}, \dot{R}_{e q}$ and $\ddot{R}_{e q}$ are all negative we can see from the equation for acoustic pressure within the gas (Eq. 3.120 in Ref. 21) that the pressure inside the bubble must simultaneously be increasing. This internal pressure increase perturbs the equilibrium relationship between bubble pressure and volume, and so the bubble will respond by ex- 
panding on a timescale commensurate with its natural response time. This pressure increase is what drives the bubble into volume oscillations.

As observed in the original paper, ${ }^{4}$ there is a small acoustic rarefaction prior to the moment of pinch-off. We hypothesize that this is due to the increasingly rapid thinning of the neck of air joining the bubble to the reservoir, causing high inward acceleration of the neck walls just prior to pinch-off. The model described here ignores everything before the moment of pinch-off, and only applies once there is a no longer a bridge of air between the new bubble and the reservoir gas.

A simple neck collapse model can be formulated by considering the neck to be composed of thin, stacked disks of air. We assume that the cone collapses without overshoot, and that the bubble has a circular flat base at all times after pinch-off. As each successive axisymmetric disk of the cone is reached by the bubble base, the surface tension energy of its edges is converted into water kinetic energy. ${ }^{22}$ We assume that the mass that is accelerated has a volume equal to the air disk it replaces and that it is accelerated to a speed $u$, which is the velocity at which the bubble base moves upwards. The assumed balance of surface tension energy and kinetic energy gives an expression for $u$ :

$$
2 \pi r \sigma \frac{1}{\cos \theta} d x=\frac{1}{2} u^{2} \pi r^{2} \rho d x,
$$

where $r$ and $x$ are defined above, $\sigma$ is the surface tension, $\rho$ is the fluid density and $\theta$ is the half-angle of the cone (see Fig. 4). Equation (2) can be rearranged and integrated to get expressions for the time taken for the base of the bubble to recede a distance $x$ from the pinch-off point:

$$
\begin{aligned}
u= & \sqrt{\frac{4 \sigma}{\rho r_{o}}} \text { if } \quad x<x_{o} \\
u= & \sqrt{\frac{4 \sigma}{\rho x \cos \theta}} \text { if } x \geq x_{o} \\
t= & \sqrt{\frac{\rho r_{o}}{4 \sigma} x_{o}+\frac{2}{3 \tan \theta} \sqrt{\frac{\rho \cos \theta}{4 \sigma}}\left\{\left[\left(x-x_{o}\right) \tan \theta+r_{o}\right]^{3 / 2}\right.} \\
& \left.-r_{o}^{3 / 2}\right\}
\end{aligned}
$$

where $t$ is the time after bubble detachment, and $r_{o}$ and $x_{o}$ are the radius and length respectively of the cylindrical thread.

Even this is an oversimplification, because of course the surface does not vanish completely. Strictly, following the model, the surface area should be reduced only by the difference between the sloped surface area on the side of the disk and the flat horizontal surface which remains when the disk has zero thickness. However, the bubble also reduces in radius slightly above the collapsed region (see Fig. 3), also providing some surface energy, and to a first approximation it seems that one cancels the other out.

Now the expression for the change of $t$ with $x$ can be used to estimate the volume decrease with time. This is given by

$$
V(t)=\pi r_{o}^{2} x_{o}+\frac{3 \pi \sigma \tan \theta}{\rho \cos \theta}\left(t-t_{o}\right)^{2},
$$

where $t_{o} \approx x_{o} \sqrt{\rho r_{o} / 4 \sigma}$. The first term accounts for the small volume of the cylindrical part of the neck, and is of very limited significance, but it is included here for consistency. The forcing timescale relevant in our study is $500 \mu \mathrm{s}$ and $t_{o}$ is of the order of $15 \mu \mathrm{s}$. It is a reasonable approximation to assume that the volume decrease with time after detachment is proportional to $t^{2}$.

The surface tension-driven decrease in volume decreases the equivalent spherical bubble radius and has two effects. It causes an acoustic rarefaction to propagate outward into the fluid, and increases the pressure inside the bubble. This internal pressure change then disturbs the equilibrium between pressure inside and outside the bubble and drives "breathing mode" radial bubble oscillations. As discussed in Deane and Czerski, ${ }^{4}$ knowledge of the volume change with time allows calculation of that forcing function and therefore also the change in bubble radius with time and the emitted sound. The details of the acoustic analysis are discussed in that paper, but we include a summary here to set the context for the model of bubble pinch-off dynamics.

The acoustic forcing term from the neck collapse is included in the Rayleigh-Plesset equation, and this was discussed in detail in the earlier paper. Equation (5) from that paper is reproduced below to show where the forcing term from the neck collapse mechanism is introduced into this equation. This linearized, harmonic form of the equation and the damping terms in it were derived by Prosperetti. ${ }^{25}$

$$
\begin{aligned}
\frac{\partial^{2} \varepsilon}{\partial t^{2}}+ & {\left[\frac{4\left(\mu+\mu_{\mathrm{th}}\right)}{\rho R_{0}^{2}}+\frac{k R_{0}}{1+k^{2} R_{0}^{2}} \omega\right] \frac{\partial \varepsilon}{\partial t}+\left[\frac{3 \kappa p_{i n, 0}}{\rho R_{0}^{2}}-\frac{2 \sigma}{\rho R_{0}^{3}}\right.} \\
& \left.+\frac{k^{2} R_{0}^{2}}{1+k^{2} R_{0}^{2}} \omega^{2}\right] \varepsilon=f(t),
\end{aligned}
$$

Where $\varepsilon$ is the fractional change in radius, $R_{o}$ is the equilibrium bubble radius, $\mu$ is the fluid viscosity, $\mu_{\text {th }}$ represents the thermal damping, $k$ is the wavenumber and $\omega$ is the angular frequency of the bubble at or close to its natural frequency, $P_{o}$ is ambient pressure and $f(t)$ is the forcing from neck collapse and jet intrusion. The Rayleigh-Plesset equation assumes a spherically symmetric bubble, but it seems that small deviations from sphericity do not affect the outcome significantly. ${ }^{4}$

The relationship between the forcing function $f$ and the fraction volume decrease $\Delta V(t) / V$ is shown in Eq. (8):

$$
f(t)=\frac{-\kappa P_{o} \Delta V(t)}{V_{o} \rho R_{0}^{2}},
$$

where $\Delta V(t)$ is the change in volume as a function of time, caused here by the neck collapse and jet intrusion. The focus of this paper is the mechanics of the collapse process which causes $\Delta V$, rather than the details of the acoustic calculation, but the equations above show the importance of the details of the bubble dynamics to the acoustic emission.

H. Czerski and G. B. Deane: Acoustic excitation of bubbles 


\section{TESTING THE MODEL AS THE SURFACE TENSION VARIES}

In order to test the predicted dependence of neck collapse on surface tension, experiments were carried out using water containing varying amounts of the surfactant Triton$\mathrm{X} 100$ to vary the surface tension. The expected value for the surface tension of pure water is $0.072 \mathrm{~N} / \mathrm{m}$ at laboratory temperatures, but we measured the value to be $0.069 \mathrm{~N} / \mathrm{m}$, and we use this here for a fair comparison with the other samples. The surfactant mixtures used had surface tension values of $0.048,0.051$ and $0.039 \pm 0.002 \mathrm{~N} / \mathrm{m}$. The value of the surface tension was measured using the capillary rise method with ten measurements made for each mixture.

Varying the surface tension has two main effects. In fluids with lower surface tension, the bubbles produced by a nozzle are smaller because the buoyancy overcomes the surface tension holding the bubble at the nozzle at a smaller volume. In practice, the bubble sizes varied from $3.5 \mathrm{~mm}$ (for $\sigma=0.039 \mathrm{~N} / \mathrm{m}$ ) to $4.1 \mathrm{~mm}$ diameter (for fresh water). In addition, the available energy from surface tension per unit area is lower, so there is a decrease in the speed at which the neck retracts.

As long as the bubbles were produced slowly, the bubble shapes were self-similar for all values of surface tension. The ratio of bubble diameter to height (distance from the pinchoff point to the top of the bubble) was checked for each set of results and was found to be the same to within $1 \%$. If the bubble production rate was increased so that the height/ diameter ratio changed by even a few percent, the neck retraction dynamics were significantly different. At higher bubble production rates, the ratio of height to diameter decreased considerably and the bubbles became much larger. As long as the interval between bubbles was more than $2 \mathrm{~s}$, the bubbling rate did not affect the results.

The bubble maximum horizontal diameter $D$ varies with $\sigma$ in a way that is consistent with the Longuet-Higgins' $\operatorname{suggestion}^{12}$ that $\sqrt{\sigma} / D$ should be constant. The neck length seems to have a very small dependence on surface tension, increasing by less than $10 \%$ as $\sigma$ decreased from $0.069 \mathrm{~N} / \mathrm{m}$ to $0.039 \mathrm{~N} / \mathrm{m}$. To model each data set, the value of $x_{o}$ used was half the total neck length measured as shown in Fig. 4 and $r_{o}$ was fixed at $4.5 \mu \mathrm{m}$. Our photographic time resolution is insufficient to accurately measure the neck width, but this is our best estimate.

Figure 5 shows a comparison of the $\sigma$ values from direct measurements and the values inferred from the bubble dynamics. There is reasonable agreement between these independent measurements of surface tension. This gives us confidence that the surface tension is the dominant influence in this experiment, and that this simple model is sufficient to estimate the behavior of the neck with time.

Figure 6 shows double strobe images of one bubble in fresh water and one in water with the lowest surface tension used here. The time between exposures is very similar for both and we note two things. First, the neck length and shape is very similar in the two cases, even though the bubbles are very different sizes. Second, the geometry of the collapse is very similar, providing evidence that the neck collapse is a

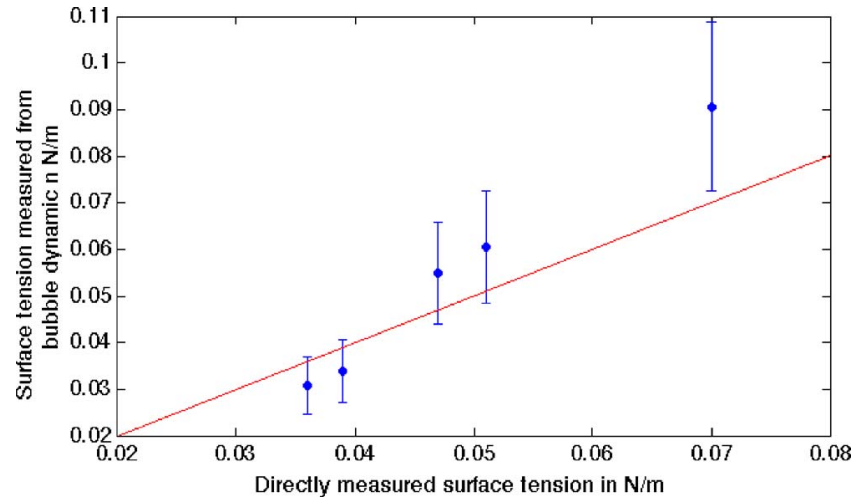

FIG. 5. (Color online) A comparison of the surface tension calculated directly and the surface tension inferred from the bubble dynamics. If the short cylindrical part of the neck is ignored, we expect $\mathrm{t}$ to be proportional to $x^{3 / 2}$. By measuring the slope of this line, we can get an estimate for $\sigma$ which can be compared with the directly-measured value. The straight line represents $1: 1$ correlation.

local phenomenon, not affected by the details of the rest of the bubble. The only significant difference visible in the photos is that the neck collapse is slower when the surface tension is lower, as expected.

\section{A. Seawater}

Bubbles are known to behave differently in seawater and fresh water. The greater number of small bubbles underneath ocean waves is commonly attributed to the reduced probability of coalescence. ${ }^{26}$ The amplitude and damping of the acoustic signals produced are also different in the two cases. ${ }^{27}$ As we are interested only in the volume change causing acoustic excitation, such effects are not the focus here. However, the ultimate application of this research is to bubbles underneath breaking waves on the open ocean and

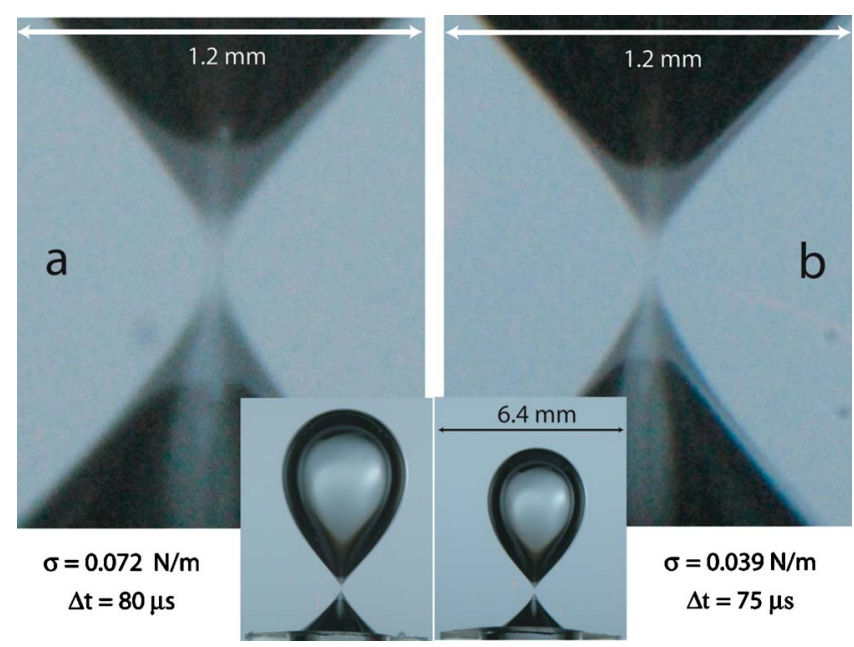

FIG. 6. (Color online) Comparison of the details of the neck at pinch-off and after a short time for $\sigma=0.072 \mathrm{~N} / \mathrm{m}$ and $\sigma=0.039 \mathrm{~N} / \mathrm{m}$. Even though the surface tension in the left-hand image (a) is almost double that for the right hand image (b), the neck shape just before detachment is a very similar length in the two cases. The difference in surface tension produces the bubble size difference shown in the insets, which both have the same scale. At this point, the collapse is only dependent on the local shape and fluid parameters, and the size and shape of the rest of the bubble does not affect the dynamics. 


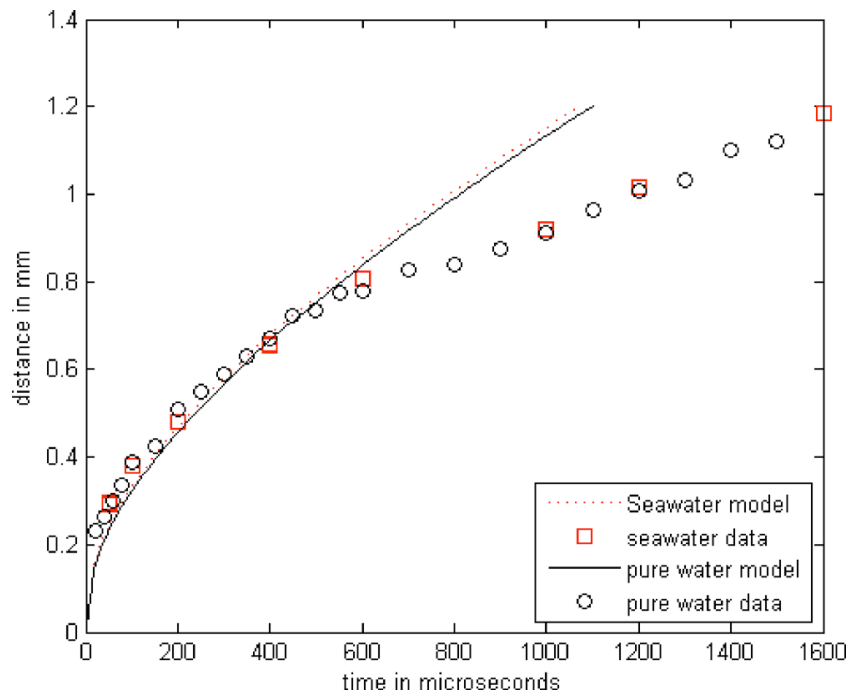

FIG. 7. (Color online) A plot of the distance from the bubble base with time for fresh and salt water. Circles represent fresh water data points and squares represent salt water. Within our experimental uncertainty, no difference can be seen in the retraction dynamics between bubbles in fresh and salt water.

so we repeated our experiments with salt water. We found that in the same experimental set-up, the shapes and sizes of the bubbles produced in seawater and fresh water were indistinguishable within our experimental uncertainty. These two cases are compared to the model in Fig. 7. The seawater used was natural filtered seawater, pumped from the La Jolla Shores beach.

Salt water has slightly higher surface tension then pure fresh water (the ideal value is 0.074 instead of $0.072 \mathrm{~N} / \mathrm{m}$ ), although we did not measure its surface tension directly here. However, it also has a higher density (typically $1027 \mathrm{~kg} \mathrm{~m}^{-3}$ ) and our model predicts that these two effects will almost cancel out since the distance of the base of the bubble from the pinch-off point varies as $\{\sigma / \rho\}^{1 / 3}$ The correction factor for seawater is therefore less than $10^{-3}$.

\section{INTERNAL JET}

As the neck collapses, a jet of water is formed inside the bubble. It has been shown ${ }^{4}$ that the volume decrease of the bubble as the neck collapses forces the bubble to begin breathing mode oscillations which cause sound radiation. In that first paper, the additional volume change due to the formation of the jet was ignored, but we examine it further here. It should be noted that there is a significant difference in jet size and behavior as the bubble production rate changes. The jet has been followed using high-speed photography in the past, ${ }^{28}$ but in these cases air was forced through a nozzle to produce bubbles at high rates (rapid sparging) and the jets were far larger. Bubbles which are grown and released slowly produce a much smaller jet which is harder to photograph. Without appropriate lighting, the jet produced by slow bubble pinch-off is obscured.

The jet was photographed using carefully placed light sources and mirrors and both strobe lights were fired simultaneously. To see the jet clearly, the bubble needs to be lit with diffuse light, and a significant proportion of that light needs to originate underneath the bubble. For bubbles illu-
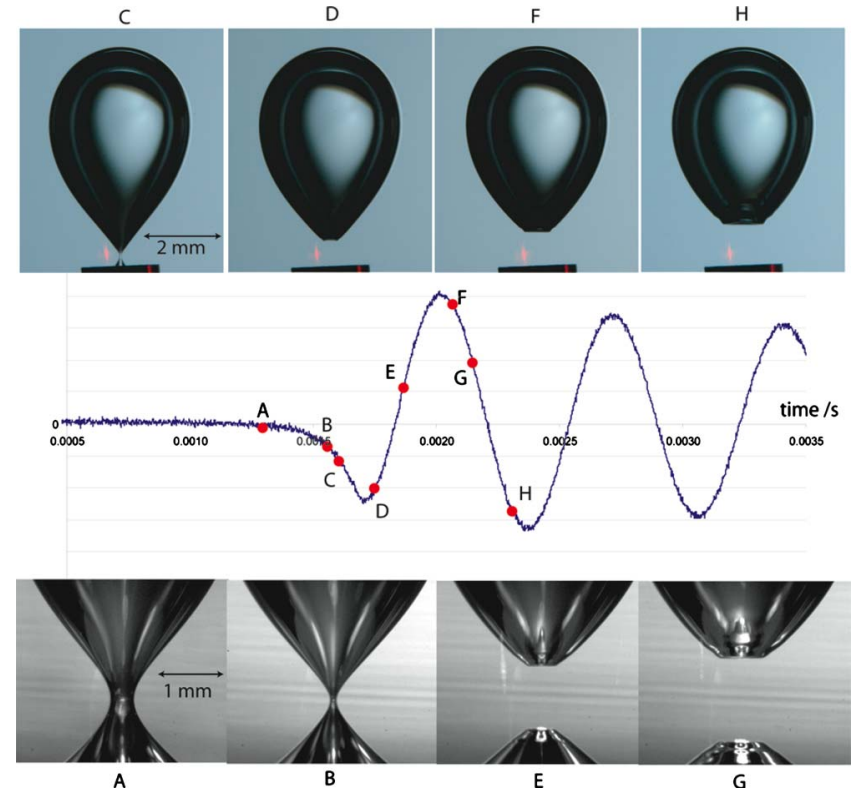

FIG. 8. (Color online) A composite diagram showing the shape of the bubble at different times during the acoustic signal. The pictures at the top are all silhouettes and the ones at the bottom are all close-ups of the neck region. The letters for each photograph correspond to the shown times on the acoustic trace. The intruding jet can be seen in $\mathrm{E}$ and $\mathrm{F}$.

minated from the back or the top, almost all the light scattered by the jet is refracted away from the camera, producing dark regions on the images. Each image could be linked directly to time on the acoustic trace, so that a series of images from different bubbles can be compared with the changes in acoustic pressure as time passes. This is shown in Fig. 8. We consider the volume of the collapsed conical region and the jet volume separately. The first part is the volume of the collapsed cone only, assuming that it collapses to produce a flat surface. The second part is the intruding jet above this imaginary surface, considered to be the bubble base (which is moving upwards with time). All the measurements shown here were made in fresh water.

The sloping bubble walls distorted the jet image because of the difference in refractive index between air and water. This was corrected for by measuring the jet profile from the photos and then adjusting each point to allow for the wall refraction. We assumed that the bubble walls were conical, and used the measured diameter at the height of each point and the known cone angle. Capillary waves propagating upwards from the pinch-off point could potentially complicate this correction, but no capillary waves are evident on the photographs until $2 \mathrm{~ms}$ after pinch off. The majority of the volume forcing happens much earlier than this, and by the time the capillary waves do appear, the jet protrudes far above the height reached by these waves, so we ignore this complication here. Jet profile positions were corrected in both the vertical and horizontal directions to produce profiles with time as shown in Fig. 9. We used seven photos of the jet at different times after pinch-off, taken in identical experimental conditions. For each one, the apparent width of the jet was recorded at several heights above the bubble base. These were halved to get the jet radius as a function of height and then each pair of coordinates was corrected for refraction. 


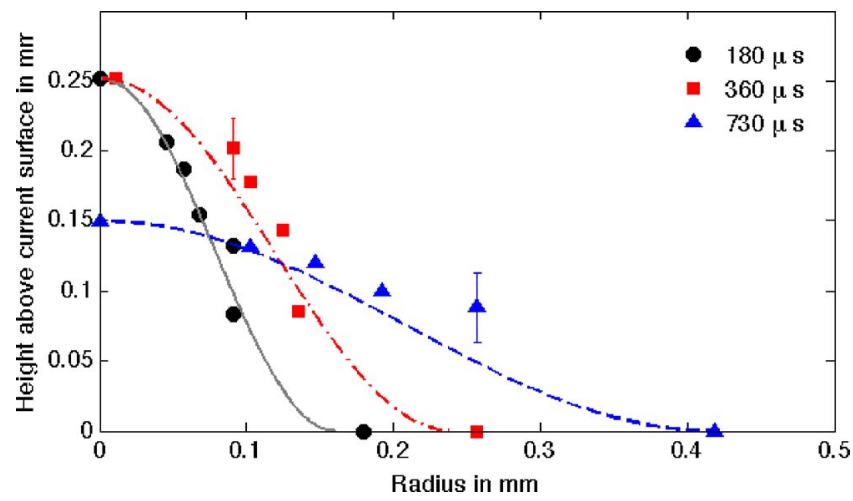

FIG. 9. (Color online) Examples of the experimental measurements of the jet profile and how the fitted jet shapes evolve with time. Cosine squared shapes were found to fit the measured jet shapes reasonably well, and selected fits are shown here. The symbols are experimentally measured points from the jet $180 \mu \mathrm{s}, 360 \mu \mathrm{s}$, and $730 \mu \mathrm{s}$ after pinch off, for comparison. The other profiles were omitted for clarity. Most of the uncertainty in the measurement comes from the estimation of the bubble wall angle, and the uncertainty increases as the radius increases. Sample error bars are shown on two points.

The final point of each series was the known base radius of the bubble on that image. Each set of jet profile points was then fitted with a cosine squared function, which provided a convenient parameterization of the jet shape. This fitted profile was used to estimate the volume of the jet corresponding to each photograph. The accuracy of the measured profiles is limited because the correction depends critically on the angle of the bubble wall at the appropriate height in the bubble, but the "conical" parts of the bubble wall move radially inwards (see Fig. 3) as the collapse progresses. Measuring this angle from our images is challenging. Nevertheless, we believe that the fitted jet profiles are a reasonable estimation of the real jet shape with time.

Figure 10 shows the decreasing importance of the jet volume as the collapse progresses. The ratio of the volume of the jet to the volume of the cone is plotted with time. Although at early times the total volume decrease with the jet included is almost twice the volume from the cone collapse alone, it quickly become less significant. The majority of the
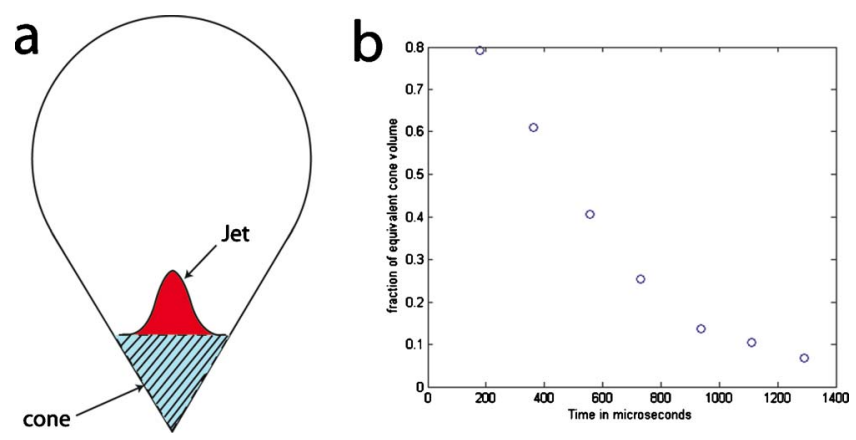

FIG. 10. (Color online) A schematic diagram of the ratio of jet volume to collapsed cone volume. (a) The volume lost according to our analytical model (the cone) and the jet volume. The dividing surface is the bubble base for the purposes of this model. (b) The ratio of the volume of the jet and the volume of the cone plotted with time. At very early times, the jet volume is almost as high as the cone volume, and therefore the volume decrease of the bubble is almost double that predicted by our simple model. At later times, the jet volume is far less significant.

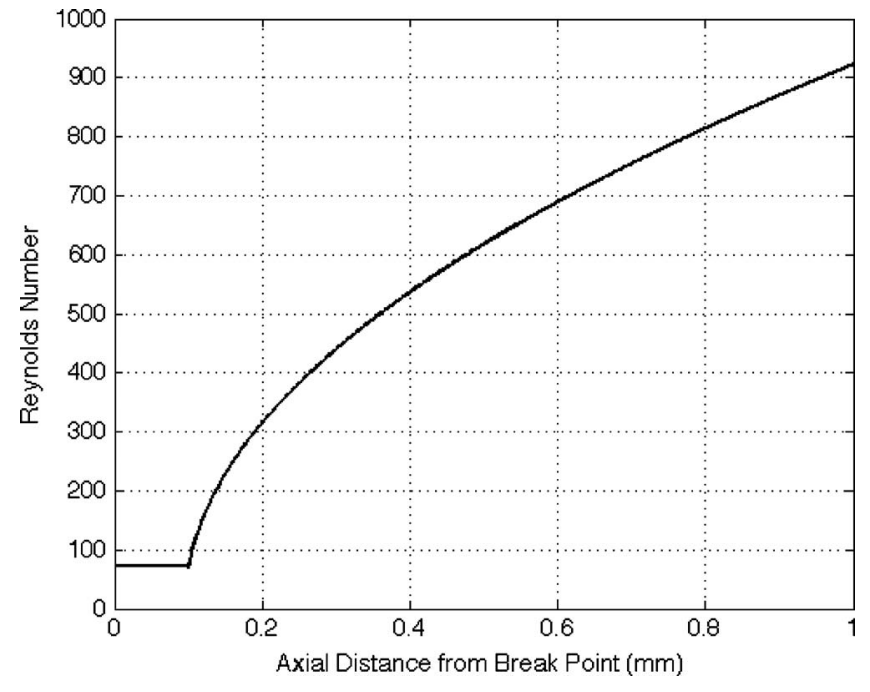

FIG. 11. The Reynolds number of the fluid flow at the neck boundary with distance from the pinch-off point. This calculation was carried out for a 2 $\mathrm{mm}$ radius bubble, using the velocity and neck width from our model.

forcing occurs in the first $200 \mu$ s after detachment, ${ }^{4}$ and the jet volume is approximately 0.8 of the corresponding cone volume at this time. It therefore seems that the additional volume of the jet intruding into the bubble almost doubles the bubble volume decrease during this critical forcing period. However, we emphasize that the collapse of the neck of air makes a larger contribution to the volume forcing than the internal jet in these experiments.

\section{DISCUSSION}

\section{A. Fluid dynamical context}

The model described in this paper is based only on the dynamics of the boundary between the air and the water. We will now briefly consider the fluid flow associated with the neck collapse, in order to examine the possible implications of that flow for our results. Figure 11 shows how the Reynolds number associated with the collapsing neck varies with distance from the pinch-off point. We calculated this using the neck collapse velocity given by Eqs. (3) and (4), and using the diameter of the neck remnant with time as the relevant fluid length scale. Close to the point of pinch-off, the Reynolds number is approximately 70 and it then increases rapidly with distance from the pinch-off point. This suggests that the fluid flow is inertially controlled, and that viscous forces can be safely neglected. The Weber number (the ratio of inertial to surface tension effects) is constant as the neck collapses, and it has a value of approximately 8 for a neck half-angle of 40 degrees The invariance of the Weber number with time follows from the fact that the diameter of the base of the neck is approximately proportional to $u^{2}$. A Weber number value of 8 is approximately the critical Weber number for the transition between jet and drop formation, and this is consistent with our observations. For slowlygrown bubbles like the ones discussed in this paper, the internal jet never broke up into drops, but when the bubbles were rapidly sparged, droplets were often seen breaking off 
the jet. However, for the bubbles studied here, surface tension is important and dominates the effects of inertia, consistent with our approach.

The maximum velocities predicted by our model are of the order of $10 \mathrm{~m} / \mathrm{s}$, far smaller than the speed of sound (approximately $1500 \mathrm{~m} / \mathrm{s}$ in water), justifying our neglect of variations in fluid density during neck collapse.

\section{B. Implications for fragmentation acoustics}

The neck collapse is very similar for fresh and salt water, since the associated changes in density and surface tension almost cancel each other out. Presumably the driving mechanism for breathing mode oscillations is almost identical in these two cases. This is important because bubbles under breaking waves are of most interest, and we conclude that the results presented here for fresh water bubble dynamics are likely to apply to seawater as well.

Manasseh et al. ${ }^{10}$ also took high-speed photographs of bubbles produced at a nozzle and related these to the acoustic trace. Their bubbles were being produced at a far higher rate $(14 \mathrm{~Hz}$, whereas ours were produced at $0.1-0.3 \mathrm{~Hz})$ and therefore had a different shape and size. They will have produced an acoustic signal with a far higher amplitude than slowly grown bubbles like the ones we studied here. However, their observed relationship between the progression of bubble shapes and the acoustic pulse is similar to what we see. Pinch-off occurs just after the rise in acoustic pressure has started, and the majority of the forcing is coincident with the neck retraction in the first $500 \mu$ s after pinch-off.

For a bubble grown slowly from a nozzle, the volume intrusion by the jet (and therefore the possible acoustic forcing that this could cause) is limited by the energy available to provide the new jet surface. Even if there were no viscous or thermal losses, the jet volume can only be less than or comparable to the volume of the cone that has collapsed up to that point, because the only energy available is the excess surface energy of the original cone. Therefore, in a slowlygrown bubble, the maximum possible volume forcing by the neck collapse and the jet together is twice that due to the neck collapse alone. In bubbles fragmenting in turbulence, there may be more energy available from the kinetic energy of the water outside the bubble, and it is conceivable that the jet could play a greater role in acoustic forcing.

Bubble pinch-off from a nozzle is expected to have the lowest possible energy for neck retraction dynamics. There is very little associated kinetic energy at the moment of pinchoff, so the only significant energy source is the surface tension energy. In other environments, the acoustic amplitude could be affected by nearby surfaces (including other bubbles) and the energy available for fragmentation. Damping will depend on the reverberant environment ${ }^{19}$ and also on the salt content. ${ }^{27}$ We have examined the features of an artificial system, but it is reasonable to assume that the same fundamental forcing mechanism may dominate when bubbles fragment in other environments.

Bubbles which are highly distorted by the surrounding flow may split into two daughter bubbles, which may then be distorted and fragment in turn. There is evidence ${ }^{29}$ that sym- metry in the neck joining the two new bubbles just before pinch-off is responsible for approximately equal energy partition between the two new bubbles after detachment. The amount of this energy varies greatly from one pair to another, which may be due to the inherent variability in the neck of air which joins the new bubbles just prior to separation. Differences in acoustic forcing caused by variation in the shape details as bubbles fragment in many environments could explain features of the resulting acoustic signals that are not yet understood.

\section{Possible further implications for oceanic noise}

The ultimate aim of this research is to understand better the natural sound produced by breaking waves, and to improve our ability to interpret that sound to infer the bubble population present. Bubble fragmentation is only one of the sound-producing events in this complicated environment. Coalescence (although inhibited in seawater), collective oscillations and pinch-off at the surface due to falling drops are also known to produce sound, and there may be other bubble events which also contribute, such as Mesler entrainment.

Leighton et al., ${ }^{30}$ and Manasseh and co-workers ${ }^{31,32}$ have investigated sound production on bubble coalescence. Manasseh found that the start of the acoustic signal is coincident with the moment of coalescence, and that the amplitude of the acoustic signal depends on the radius of the smaller bubble. They also noted that the acoustic signal always starts with a compression. Manasseh also investigated possible excitation mechanisms and found that an instantaneous pressure change associated with the different Laplace pressure correctly predicted the scaling of the pulse amplitude with small bubble radius, but underpredicted the magnitude by a factor of approximately 16. Numerical model calculation of coalescence correctly predicted the amplitude of the acoustic signal, but did not isolate a specific physical mechanism for the bubble excitation. We note here that both bubble pinch-off and coalescence events have a common geometrical feature. The neck collapse during pinch-off is rapid because the free surfaces have a very small radius of curvature, leading to high acceleration of water away from the pinch-off point. In the case of coalescence, the small radius of curvature is present around the inside of the annulus of water surrounding the point of coalescence, forcing the water to undergo rapid outward radial acceleration. This similarity is suggestive and this might be a candidate mechanism for the excitation of coalescing bubbles.

\section{CONCLUSIONS}

We used bubbles emerging slowly from a nozzle to test a simple analytical model for the collapse of the bubble neck in fluids with different surface tension. Ideally, the acoustic traces from these bubbles would also be used to calculate the forcing function to compare with the volume forcing model, as was done in the previous paper. However, the experiments that produced the data shown in Fig. 5 came from a very small tank, and while this makes detailed photography possible, reverberation from the tank walls may affect the magnitude of the acoustic signal significantly. This is why we 
have not used the acoustic signals from the small tank with surfactant to directly calculate the magnitude of the forcing function.

The model performs remarkably well, accounting for the main features and the overall magnitude of the effect of varying surface tension. We conclude that for an approximate calculation of the dynamics, the bulk of the bubble can be ignored; neck collapse is a local phenomenon only, dependent on the surface tension and the shape of the neck but not on the rest of the bubble.

In the first $100 \mu$ s after pinch-off, the volume of the jet intruding into the bubble is very similar in magnitude to the total volume of the collapsed conical neck segment. The early volume forcing is therefore almost twice that predicted by the simple neck collapse model alone. As time passes, the jet volume decreases steadily compared to the cone volume, and so its importance in the volume forcing of the breathing mode oscillations of the bubble will also decrease. To take the jet into account, the volume decrease predicted by our neck collapse model should be multiplied by a factor that is close to 2 at very early times and decreases monotonically with time thereafter. After $500 \mu$ s (the timescale for which our neck collapse model matches the data), the total contribution of the jet to acoustic forcing is an additional $35 \%$. The intruding jet volume is not the dominant contribution to the forcing although it may have a noticeable effect at very early times in the collapse.

Our results confirm the dominant features of the neck collapse just after a new bubble is formed at a nozzle. The associated volume change has previously been shown to account for the forcing necessary to drive the bubble into breathing mode oscillations which then produce a pulse of sound. We have presented further evidence to support the simple analysis outlined in Deane and Czerski, ${ }^{4}$ and some of the issues outstanding from that paper have been addressed. Neck collapse as a new bubble is formed is a common feature in many environments. Consequently, we expect this new understanding of the origin of the acoustic pulse to have implications for the sound production caused by any new bubble.

\section{ACKNOWLEDGMENTS}

We thank Dr. Dale Stokes, Mr. Cary Humphries and Mr. James Uyloan for assistance with data collection. We acknowledge financial support from the National Science Foundation Physical Oceanography Program, OCE 07-27140 and the Office of Naval Research Ocean Acoustics Program N00014-07-1-1030.

${ }^{1}$ T. G. Leighton and A. J. Walton, "An experimental study of the sound emitted from bubbles in a liquid," Eur. J. Phys. 8, 98-104 (1987).

${ }^{2} \mathrm{H}$. Medwin and A. C. Daniel, "Acoustical measurements of bubble production by spilling breakers," J. Acoust. Soc. Am. 88, 408-412 (1990).

${ }^{3}$ H. C. Pumphrey and P. A. Elmore, "The entrainment of bubbles by drop impacts," J. Fluid Mech. 220, 539-567 (1990).

${ }^{4}$ G. B. Deane and H. Czerski, "A mechanism stimulating sound production from air bubbles released from a nozzle," J. Acoust. Soc. Am. EL126, 123
(2008)

${ }^{5} \mathrm{M}$. Longuet-Higgins, "An analytical model of sound production by raindrops," J. Fluid Mech. 214, 395-410 (1990).

${ }^{6}$ H. C. Pumphrey and J. E. Ffowcs Williams, "Bubbles as sources of ambient noise," IEEE J. Ocean. Eng. 15, 268-274 (1990).

${ }^{7}$ M. S. Longuet-Higgins, "Monopole emission of sound by asymmetric bubble oscillations. Part I. Normal modes," J. Fluid Mech. 201, 525-541 (1989).

${ }^{8}$ M. S. Longuet-Higgins, "Monopole emission of sound by asymmetric bubble oscillations. Part II. An initial value problem," J. Fluid Mech. 201, 543-565 (1989).

${ }^{9}$ R. D. Hollett and R. M. Heitmeyer, "Noise generation by bubbles formed in breaking waves," Sea Surface Sound (Kluwer Academic, Boston, 1988), pp. 449-461.

${ }^{10}$ R. Manasseh, S. Yoshida, and M. Rudman, "Bubble formation processes and bubble acoustic signals," Proceedings of the 3rd International Conference on Multiphase Flow, Lyon, France (1998), Paper No. 202.

${ }^{11}$ R. Manasseh, R. F. LaFontaine, J. Davy, I. Shepard, and Y. G. Zhu, "Passive acoustic bubble sizing in sparged systems," Exp. Fluids 30, 672-682 (2001).

${ }^{12}$ M. S. Longuet-Higgins, B. R. Kerman, and K. Lunde, "The release of air bubbles from an underwater nozzle," J. Fluid Mech. 230, 365-390 (1991).

${ }^{13}$ G. B. Deane and M. D. Stokes, "Scale dependence of bubble creation mechanisms in breaking waves," Nature (London) 418, 839-844 (2002).

${ }^{14}$ J. M. Gordillo, A. Sevilla, J. Rodriguez-Rodriguez, and C. MartinezBazan, "Axisymmetric bubble pinch-off at high Reynolds numbers," Phys. Rev. Lett. 95, 194501 (2005).

${ }^{15}$ J. C. Burton, R. Waldrep, and P. Taborek, "Scaling and instabilities in bubble pinch-off," Phys. Rev. Lett. 94, 184502 (2005).

${ }^{16} \mathrm{~S}$. T. Thoroddsen, T. G. Etoh, and K. Takehara, "Experiments on bubble pinch-off," Phys. Fluids 19, 042101 (2007).

${ }^{17}$ H. N. Oguz and A. Prosperetti, "Dynamics of bubble growth and detachment from a nozzle," J. Fluid Mech. 257, 111-145 (1993).

${ }^{18}$ P. A. Elmore, G. L. Chahine, and H. N. Oguz, "Cavity and flow measurements of reproducible bubble entrainment following drop impacts," Exp. Fluids 31, 664-673 (2001).

${ }^{19}$ T. G. Leighton, P. R. White, C. L. Morley, and J. W. L. Clarke, "The effect of reverberation on the damping of bubbles," J. Acoust. Soc. Am. 112, 1366-1376 (2002).

${ }^{20}$ M. Strasberg, "The pulsation frequency of nonspherical gas bubbles in liquids," J. Acoust. Soc. Am. 25, 536-537 (1953).

${ }^{21}$ T. G. Leighton, The Acoustic Bubble (Academic, New York, 1994), p. 155.

${ }^{22}$ The energy balance analysis was motivated by physically interpreting a scaling law from a numerical study (Ref. 23) and a subsequent experimental paper (Ref. 24), which considered the collapse of air disks formed underneath impacting rain drops.

${ }^{23} \mathrm{H}$. N. Oguz and A. Prosperetti, "Bubble oscillations in the vicinity of a nearly plane free surface," J. Acoust. Soc. Am. 87, 2085-2092 (1990).

${ }^{24}$ S. T. Thoroddsen, T. G. Etoh, and K. Takehara, "Air entrapment under an impacting drop," J. Fluid Mech. 478, 125-134 (2003).

${ }^{25}$ A. Prosperetti, "Thermal effects and damping mechanisms in the forced radial oscillations of gas bubbles in liquids," J. Acoust. Soc. Am. 61, 17-27 (1977).

${ }^{26}$ V. S. J. Craig, B. W. Ninham, and R. M. Pashley, "The effect of electrolytes on bubble coalescence in water," J. Phys. Chem. 97, 10192-10197 (1993).

${ }^{27}$ A. R. Kolaini, "Sound radiation by various types of laboratory breaking waves in fresh and salt water," J. Acoust. Soc. Am. 103, 300-307 (1998).

${ }^{28}$ R. Manasseh and T. Nicholls, "Album of visualization," The Visualization Society of Japan 13, 25-26 (1996).

${ }^{29}$ G. B. Deane and M. D. Stokes, "The acoustic excitation of bubbles fragmenting in sheared flow," J. Acoust. Soc. Am. 124, 3450-3463 (2008).

${ }^{30}$ T. G. Leighton, K. Fagan, and J. E. Field, "Acoustic and photographic studies of injected bubbles,” Eur. J. Phys. 12, 77 (1991).

${ }^{31}$ R. Manasseh, G. Riboux, A. Bui, and F. Risso, "Sound emission on bubble coalescence: Imaging, acoustic and numerical experiments," Proceedings of the 16th Australian Fluid Mechanics Conference (2007), pp. 167-173.

${ }^{32}$ R. Manasseh, G. Riboux, and F. Risso, "Sound generation on bubble coalescence following detachment," Int. J. Multiphase Flow 34, 938-949 (2008). 